\title{
Real-time Image Processing and Restoration Prototype for the SEM: Initial Steps
}

\author{
G. Chilton ${ }^{1}$, B.C. Breton², D.M. Holburn² and N.H.M. Caldwell ${ }^{3}$
}

${ }^{1}$ BT plc, Adastral Park, Martlesham Heath, Ipswich, United Kingdom

2. Department of Engineering, University of Cambridge, Cambridge, United Kingdom

3. School of Science, Technology and Engineering, University of Suffolk, Ipswich, United Kingdom

In previous work, we have reported on a series of projects utilizing GPGPUs (General Purpose Graphics Processing Units) to improve instrument performance, notably preliminary work in scanning electron microscope (SEM) autofocusing [1], autofocusing and astigmatism correction using Fourier domain algorithms [2], specific SEM-related image processing techniques [3], and automated gun alignment for tungsten filament emission sources [4]. GPGPU computation is now an established means of exploiting the tens to thousands of processor cores available on modern graphics cards, with the proprietary Compute Unified Device Architecture introduced by NVIDIA in 2006 enabling developers to utilize GPUs by means of extensions to C and similar programming languages (see [5]), and OpenCL (Open Compute Language) providing an alternative open-source framework for executing parallel programs across heterogeneous hardware.

Although electron-optical advances and higher brightness electron sources have continued to improve SEM resolution, these necessitate more complex instrument parameter configurations, higher operator skill levels, and hardware and running costs. There has been recent research on using point spread function (PSF) deconvolution as an image restoration technique for the SEM, where an SEM image can be restored to more accurately depict what is being imaged through the deconvolution of the PSF and the image [6, $7,8]$. Real-time image processing and image restoration techniques enabled through GPGPU computation offer the potential to improve SEM images on all instruments, both in terms of improving upon what is attainable through the available hardware and enhancing what is achievable by microscope operators (without further adjustment of instrument parameters). This has motivated the present work to design and implement a toolbox for real-time image improvement.

The microscope employed for development and testing was a Carl Zeiss 1430VP SEM, which had been retrofitted with a very low specification NVIDIA GeForce 8600 GT graphics card with only 32 processors. A desktop computer equipped with a NVIDIA GeForce 970 graphics card (1664 processor cores) provided an opportunity to test higher performance ranges using a graphics card specification suitable for current microscopes. Access to images direct from the SEM was enabled through Carl Zeiss' Application Programming Interface to their SmartSEM ${ }^{\mathrm{TM}}$ software. The prototype was designed and implemented with a combination of Microsoft Visual Studio, OpenCL and the image processing routines available through the OpenCV library. OpenCL and OpenCV were chosen to ensure GPGPU neutrality and easier transitioning between new 64-bit development hardware and the older 32-bit SEM configuration

Figure 1 shows a current workflow from the prototype toolbox. Firstly the live SEM image is captured via a call to the SmartSEM ${ }^{\mathrm{TM}}$ API, saved to hard disc and then read by the toolbox. Secondly if required, an image denoising process can be executed to sharpen the image. This utilizes the OpenCV denoising filter with a template window size of 7 pixels, search window size of 21 pixels and a filter strength of 25 . These values were determined through experiment on high-resolution images to yield useful noise removal without noticeable detail loss. The third stage is to perform brightness and contrast optimization of the 
image. Two options are available in the prototype: a straightforward histogram equalization function and more experimental Fourier domain functions for distinct brightness and contrast optimization through adjusting the Fourier space magnitude of the image. Thus by dividing the magnitude of the image by a brightness coefficient, it is relatively easy to increase or decrease the brightness of the image. The fourth stage was to perform a sharpness optimization. This relies on image variance as the measure of sharpness. A Gaussian blur is applied to the image in the spatial domain, generating a secondary image which is then added to the original image to form a resulting image. The variance of this resulting image is then calculated to determine how much it has increased from the original starting image in this stage of the workflow. The process of blur, add, and calculate will be repeated until the variance of the final image is $30 \%$ greater than the original starting image. The 30\% threshold represents a balance between sharpening the image and enhancing image noise unacceptably. The fifth stage of the workflow was intended to utilize point-spread functions to perform additional image restoration measures to the image. At the time of writing, the point spread functions used in the prototype are too inaccurate, leading to image degradation rather than image improvement. The final image is displayed in a separate GUI distinct from the main SEM user interface. The development SEM is equipped with dual monitors, so for ease of use, the SEM interface is displayed on one screen and the prototype results on the second screen.

The prototype enhancement package remains a work in progress. Additional effort is required to rigorously test the brightness and contrast optimizations on a wider range of samples to discern sensible coefficients for a variety of situations, and further work is necessary to calculate more reliable point spread functions to undertake image restoration. Nevertheless the prototype is capable of achieving useful improvements in image quality in near real-time without requiring changes to instrument parameters [8].

\section{References:}

[1] N.H.M. Caldwell et al, Microsc. Microanal. 18 (Suppl S2) (2012), p. 1210.

[2] N.H.M. Caldwell et al, Microsc. Microanal. 19 (Suppl S2) (2013), p. 792.

[3] N.H.M. Caldwell et al, Microsc. Microanal. 19 (Suppl S2) (2013), p. 774.

[4] D.M. Holburn et al, Microsc. Microanal. 20 (Suppl 3) (2014), p. 46.

[5] J. Sanders and E. Kandrot, CUDA by Example: An Introduction to General-Purpose GPU Programming, Addison Wesley, 2010.

[6] E. Lifshin et al, Microsc. Microanal. 20 (Suppl 3) (2014) p. 386.

[7] M.C. Nevins et al, Microsc. Microanal. 23 (Suppl 1) (2017), p. 126

[8] M.D. Zotta and E. Lifshin, Microsc. Microanal. 23 (Suppl 1) (2017), p. 124

[9] NVIDIA GeForce and CUDA are trademarks of NVIDIA Corporation. SmartSEM ${ }^{\text {TM }}$ is a trademark of Carl Zeiss Microscopy. This research was supported by funding from Carl Zeiss Microscopy and the in-kind support of the first author by BT plc. The authors gratefully acknowledge the assistance of Carl Zeiss personnel, especially David Hubbard, in undertaking this research.

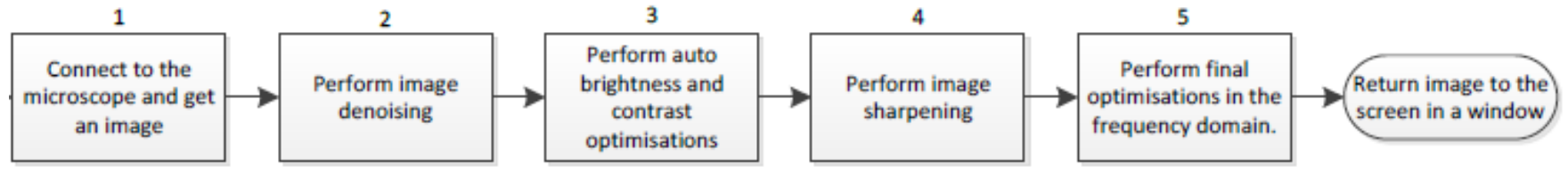

Figure 1. A Sample Workflow of the Real-time Image Improvement Toolbox 